\title{
El origen de los trastornos mentales: Un nuevo enfoque desde el estudio de la dinámica de la
} personalidad

\section{The origin of mental disorders: A new approach from the study of personality dynamics}

\author{
Salvado Amigó. \\ Departament de Personalitat, Avaluació i \\ Tractaments Psicològics \\ Universitat de València \\ Valencia, Spain \\ Salvador.Amigo@uv.es
}

\author{
Joan C. Micó. \\ Intitut Universitari de Matemàtica \\ Multidisciplinar \\ Universitat Politècnica de València \\ Valencia, Spain \\ jmico@mat.upv.es
}

\author{
Antonio Caselles. \\ IASCYS member \\ Departament de Matemàtica Aplicada. \\ Universitat de València \\ Valencia, Spain \\ Antonio.Caselles@uv.es
}

\begin{abstract}
Resumen - Cada vez hay un mayor consenso en las ciencias de la salud mental (psicología, psiquiatría) en cuanto a que no hay una línea divisoria clara entre la personalidad "normal“ y la personalidad "anormal” o los trastornos mentales. De hecho, los rasgos básicos de personalidad predisponen o se solapan con los trastornos clínicos mentales. Por ejemplo, el Neuroticismo, como factor que predispone a las neurosis, predispone a padecer trastornos de ansiedad, depresión y obsesivos. Esta cuestión sirve para presentar un resumen del trabajo de nuestro grupo (Antonio Caselles, Joan C. Micó y Salvador Amigó) y señalar futuras investigaciones. Así, tras repasar nuestros estudios sobre la dinámica del Factor General de Personalidad (FGP) y su sustrato biológico, el sistema de estrés, en respuesta a estímulos ambientales, como son las drogas, proponemos que los mismos mecanismos que habíamos encontrado, sirven para explicar el origen de los diferentes trastornos mentales, tomando como condición inicial el nivel de FGP. De esta manera, nos adentramos en la comprensión global y sistémica del individuo, que no sólo busca encontrar los mecanismos que explican el comportamiento humano, sino también los que determinan su salud o enfermedad mental.
\end{abstract}

Keywords-Factor General de Personalidad; Teoría de Sistemas; Dinámica de la Personalidad; Trastornos Mentales

\begin{abstract}
There is a growing consensus in the mental health sciences (psychology, psychiatry) that there is no clear dividing line between "normal" personality and "abnormal" personality or mental disorders. In fact, basic personality traits predispose or overlap with clinical mental disorders. For example, Neuroticism, as a factor that predisposes to neurosis, predisposes to suffer from anxiety, depression and obsessive disorders. This question serves to present a summary of the work of our group (Antonio Caselles, Joan C. Micó and Salvador Amigó) and to point out future research. Thus, after reviewing our studies on the dynamics of the General Personality Factor (FGP) and its biological substratum, the stress system, in response to environmental stimuli, such as drugs, we propose that the same mechanisms that we had found, serve to explain the origin of the different mental disorders, taking the level of FGP as an initial condition. In this way, we delve into the global and systemic understanding of the individual, who not only seeks to find the mechanisms that explain human behavior, but also those that determine their health or mental illness.
\end{abstract}

Keywords-General Personality Factor; Systems Theory; Dynamics of the Personality; Mental disorders 


\section{La difícil relación entre lo normal y lo anormal en psicología y psiquiatría}

En la actualidad, existe una tendencia generalizada entre los psicólogos, psiquiatras y neurólogos, en cuanto a considerar que los trastornos mentales representan el extremo de un continuo que va desde la personalidad normal, pasando por los trastornos de personalidad, hasta llegar a los trastornos mentales. Es decir, que no existe una clara línea divisoria entre lo normal y lo patológico.

La asunción paulatina por parte del DSM (Manual Diagnóstico y Estadístico de los Trastornos Mentales) de la perspectiva dimensional de los trastornos frente a la categorial, da buena prueba de ello. Pero ya desde la perspectiva constitucionalista de la personalidad de Krestchmer y Sheldon, en la primera mitad del siglo $\mathrm{XX}$, se consideraba que no hay una discontinuidad fundamental entre lo normal y lo anormal. En época más reciente, diferentes estudios sobre la personalidad y los trastornos mentales [1] y la teoría etiológica de los trastornos de personalidad [2] abogan por esta misma idea.

Una de las teorías de la personalidad más influyentes del siglo XX, la teoría de personalidad de Eysenck, sostiene que las dimensiones de personalidad predisponen a determinados trastornos psicológicos [3]. Eysenck distingue tres dimensiones básicas de personalidad: Extraversión, que mide el grado de sociabilidad, el Neuroticismo y el Psicoticismo. Estas dos últimas dimensiones representan propensiones a padecer trastornos neuróticos (ansiedad, depresión, fobias, obsesiones, etc.) y trastornos psicóticos (a la vez que psicopáticos), respectivamente.

Si bien Eysenck insiste en que se trata de dimensiones de la "personalidad normal”, es cierto que las personas con trastornos neuróticos o psicóticos puntúan alto en las dimensiones de Neuroticismo y Psicoticismo, respectivamente. Esto puede interpretarse como que hay un solapamiento entre las dimensiones básicas de personalidad y los síndromes clínicos, que la puntuación extrema en una de estas dimensiones refleja la posible existencia de un trastorno clínico, si bien el trastorno debe diagnosticarse de manera independiente usando otro tipo de instrumento de evaluación.

\section{Dinámica de la personalidad y Factor General de Personalidad}

Los estudios anteriores citados sobre la relación entre lo normal y lo anormal en psicología son estudios transversales, correlaciónales, pero no se sugiere una dinámica que explique el surgimiento de los trastornos mentales desde los factores de personalidad básicos que subyacen a los mismos.

Este es, precisamente, uno de los objetivos actuales de nuestro grupo de investigación, compuesto por Antonio Caselles y Joan C. Micó como expertos en teoría matemática de sistemas, y el psicólogo Salvador Amigó. Desde el principio de nuestro trabajo, ya algo más de una década, nos hemos dedicado al estudio de la dinámica de la personalidad en respuesta a un estímulo ambiental, como el consumo de una droga. Para ello, hemos partido de la Teoría del Rasgo Único de Personalidad (TRUP) [4] y de la formulación matemática de sistemas complejos desde la teoría de sistemas.

Se ha elaborado un modelo matemático complejo para explicar la dinámica de la personalidad, pero buscando el cambio de la personalidad global que una droga produce. El Rasgo Único de personalidad es la Extraversión y se considera el súper-rasgo básico de personalidad que representa la personalidad completa, y que puede ilustrarse como un continuo que va desde un extremo de impulsividad y agresividad (extraversión extrema) hasta el polo opuesto de inhibición y temor (introversión extrema).

Este Rasgo Único pasó a denominarse Factor General de Personalidad (FGP), para adaptarnos a la nomenclatura aceptada internacionalmente de este enfoque de rasgo único de personalidad, que ha experimentado un auge considerable en los últimos años. Elaboramos un cuestionario para medir este súper-rasgo. Así, utilizando el Cuestionario del Factor General de Personalidad (CFGP) comprobamos que el FGP puede considerarse un súper-rasgo que engloba y representa los principales factores de los modelos de personalidad más en boga en la actualidad [5].

Si nos detenemos en la relación entre el FGP y los 3 factores del modelo de Eysenck, el resultado fue que el FGP correlaciona positiva y significativamente con Extraversión y Psicoticismo y negativamente con Neuroticismo. Así, este resultado es congruente con la TRUP, de forma que las dimensiones externalizantes (Extraversión y Psicoticismo) se situarían en un polo del continuo, mientras que las internalizantes (Neuroticismo) se situarían en el polo opuesto.

Posteriormente elaboramos una escala de 5 adjetivos que representaban el FGP, tanto en versión rasgo (general) como en versión estado (momentánea): la Escala de Cinco Adjetivos del Factor General de Personalidad (ECA-FGP) [6]. Es precisamente la versión estado la que 
nos permitió estudiar la dinámica del FGP a corto plazo en respuesta a diferentes estímulos, entre los que destacan las drogas estimulantes, como el café y el metilfenidato.

En todos estos estudios se validó la TRUP, es decir, se constató que los extravertidos se excitan más rápida y fácilmente que los introvertidos y que esta estimulación se extingue también más rápidamente [7]. Además del uso de la ECA-FGP para analizar la dinámica, también pudimos constatar el sustrato biológico de los mecanismos activadores e inhibitorios del FGP, partiendo de la base de que es el sistema de estrés el súper-sistema biológico que subyace a la dinámica del FGP [8-10].

Estos mecanismos excitatorios e inhibitorios diferenciales para extravertidos e introvertidos nos permitieron elaborar un modelo de cambio de personalidad a largo plazo, basado en los cambios en los niveles de estrés. Así, cuando se repite una dosis alta de una droga estimulante, el estrés producido tiende a recuperarse más lentamente a largo plazo, por lo que un consumidor frecuente de drogas estimulantes cambiará su personalidad desde la extraversión (que predispone al consumo de drogas) hacia la introversión (que representa una sobrecarga del sistema de estrés y explica los mecanismos de tolerancia de los efectos de drogas). Así, elaboramos un modelo de adicción a la cocaína que modelaba las consecuencias de un consumo prolongado de la droga [11].

Por tanto, habíamos sido capaces de explicar el cambio de personalidad global y completa en respuesta al consumo de drogas, tanto a corto como a largo plazo. También pudimos constatar que estos cambios se producían en los cinco grandes rasgos de personalidad del modelo de personalidad más influyente en la actualidad, el modelo de los Cinco Grandes, que propone la existencia de 5 grandes rasgos (dominios) de personalidad: Extraversión, Neuroticismo, Apertura a la Experiencia, Amabilidad y Responsabilidad [12].

No obstante estos hallazgos, que nos llevaron a explicar el origen y desarrollo de las drogodependencias, seguíamos sin contar con una explicación suficiente sobre el surgimiento de los diferentes trastornos mentales. La pregunta es: ¿cómo surgen todos los diferentes tipos de trastornos mentales, desde la depresión y ansiedad hasta la esquizofrenia y la psicopatía?

\section{La dinámica del Factor General de Personalidad en el origen de los trastornos mentales}

$\mathrm{Y}$ este es precisamente uno de los principales objetivos de nuestra investigación actual: explicar el surgimiento de los diferentes trastornos mentales a partir de la dinámica del FGP. Por ello, vamos ahora a exponer las principales ideas que estamos desarrollando en la actualidad.

Ya hemos presentado anteriormente el resultado de los análisis correlaciónales entre el FGP medido con el CFGP y los 3 factores de Eysenck, medidos con el EPQ [13]. Recordemos que el FGP correlaciona positiva y significativamente con Extraversión y Psicoticismo y negativamente con Neuroticismo.

Pues bien, podemos ya proponer una dinámica que relaciona el FGP y los 3 factores de Eysenck, considerados en realidad como 3 sub-factores del súperrasgo de personalidad que es el FGP.

La puntuación obtenida por cualquier individuo en el CFGP representa una puntuación entre el polo de Extraversión y el de Introversión. Así, ser más o menos extravertido o más o menos introvertido se considera una condición inicial de la dinámica propuesta. Los extravertidos cuentan con un sistema de estrés que responde rápido a los estímulos activadores, no dejando apenas huella, mientras que los introvertidos, con un sistema de estrés sobrecargado, responden en menor medida a los estímulos activadores y en mayor medida a los inhibidores, dejando además una huella perdurable en el sistema de estrés, sobrecargándolo todavía más y cronificando un alto nivel de estrés. Es este sistema de estrés sobrecargado el responsable de determinados trastornos clínicos, de carácter neurótico, como son la depresión, ansiedad, obsesiones, compulsiones, etc. Esto se producirá fácilmente en los introvertidos cuando se repite el estímulo, sobre todo los estímulos aversivos.

En este punto hay que darse cuenta que nuestra propuesta de un FGP se relaciona no solo con la teoría de Eysenck sino también con la teoría de la personalidad de Gray, que considera la existencia de dos grandes sistemas cerebrales y comportamentales, el sistema activador, especialmente sensible a los estímulos incentivadores, y el sistema inhibidor, especialmente sensible a los estímulos aversivos [14]. Existe una relación significativa entre los modelos de personalidad de Eysenck y Gray. Así, el sistema activador representaría la extraversión, mientras que el sistema inhibidor representaría la introversión. El Neuroticismo representa la intensidad de los dos sistemas. Y existiría un tercer sistema de lucha-huida, que se relacionaría con el Psicoticismo.

¿Qué sucede con los extravertidos, con un sistema de estrés más flexible, cuando se les somete a repetidas dosis de sustancias estimulantes como estímulos 
activadores? Pues que el sistema de estrés comienza a sobrecargarse (como en el caso de los introvertidos, pero más lentamente). Con un sistema de estrés sobrecargado y partiendo de la condición inicial de Extraversión, la reacción del sistema de estrés es intensa pero rápida a corto plazo, mientras que es más lenta y poco flexible a largo plazo. En el primer caso la persona presentará síntomas histéricos y reacciones psicopáticas, así como síntomas positivos de esquizofrenia, mientras que en el segundo caso, con una repetición de alta estimulación en el largo plazo, la persona presentará síntomas de tipo neurótico, como compulsiones y obsesiones. $\mathrm{Y}$ esto último es lo que se desprende de nuestro modelo de personalidad y consumo de cocaína a largo plazo [11].

En el caso de los introvertidos, si continúa la estimulación muy intensa durante mucho tiempo, debido al mecanismo de inhibición transmarginal, el sistema de estrés puede descargarse de forma que empiece a responder con conductas agresivas y síntomas psicóticos.

A continuación, en la Tabla 1 , se presenta en forma de resumen, un esquema de lo que se acaba de exponer.

Tabla 1. Trastornos según rasgos de personalidad

\begin{tabular}{|c|c|c|c|}
\hline Condición inicial & $\begin{array}{c}\text { Rasgo } \\
\text { patológico } \\
\text { predominante }\end{array}$ & $\begin{array}{c}\text { Cambio } \\
\text { corto-medio } \\
\text { plazo }\end{array}$ & $\begin{array}{c}\text { Cambio largo } \\
\text { plazo }\end{array}$ \\
\hline EXTRAVERSIÓN & Psicoticismo & $\begin{array}{c}\text { Síntomas } \\
\text { psicóticos } \\
\text { Psicopatía }\end{array}$ & $\begin{array}{c}\text { Obsesiones y } \\
\text { compulsiones } \\
\text { neuróticas }\end{array}$ \\
\hline INTROVERSIÓN & Neuroticismo & $\begin{array}{c}\text { Trastornos } \\
\text { emocionales }\end{array}$ & $\begin{array}{c}\text { Síntomas } \\
\text { psicóticos }\end{array}$ \\
& & & Agresividad \\
\hline
\end{tabular}

Para entender mejor estos cambios de personalidad a través del modelo dinámico de respuesta diseñado por los autores [7-10] para el FGP se habría de acudir a la teoría de bifurcaciones. La bifurcación en un sistema dinámico es un cambio brusco en el patrón de respuesta debido al cambio del valor de algún parámetro del modelo, generalmente, de origen estocástico o debido a un cambio del estímulo.

La bifurcación en el modelo de respuesta puede reproducirse con la introducción de términos no lineales en el efecto excitador, que hasta el momento sólo es proporcional al estímulo [7-10], o por la consideración de su variación espacio temporal, con la que es posible cuantizar alguno de los parámetros [15]. Esta última opción, que es uno de los objetivos actuales de investigación del grupo, proporciona una riqueza de respuestas mucho más amplia.

Independientemente del modelo que se considere, la bifurcación habrá de reproducir una respuesta anómala, representativa del trastorno mental. Por ejemplo, un trastorno mental muy extendido es el trastorno bipolar. Si buscamos reproducir la dinámica del trastorno bipolar, ésta ha de ser claramente oscilante. En la Figura 1 reproducimos una dinámica oscilante, en la que se alternan períodos de manía (Extraversión extrema) y depresión (Introversión extrema).

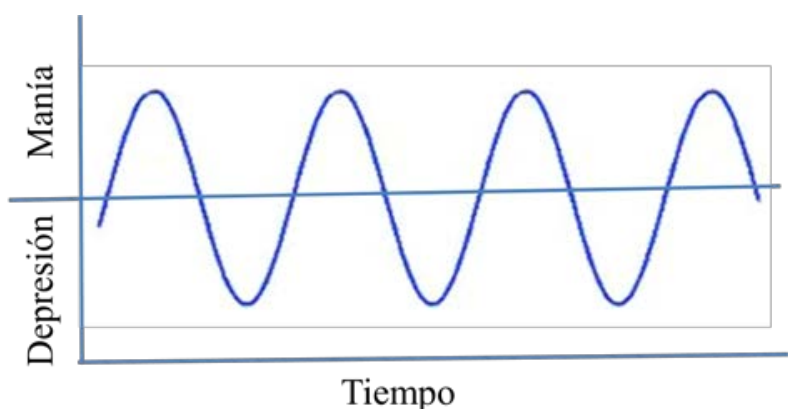

Figura 1. Modelo de alternancia de períodos de manía y depresión del trastorno bipolar.

Se ha estudiado la personalidad de los pacientes con trastorno bipolar. Desde la teoría de la personalidad de Eysenck, el Neuroticismo y Psicoticismo predicen el trastorno. También se ha podido observar que las puntuaciones altas en Neuroticismo y bajas en Extraversión distinguen a los pacientes unipolares de los bipolares [16-18].

En cuanto al estudio de la personalidad de los pacientes con trastorno bipolar desde el modelo de los Cinco Grandes, se ha observado que el Neuroticismo y la Amabilidad (negativa) predicen el trastorno. Ahora bien, si consideramos la manía y la depresión como dos dimensiones separadas, la depresión se asocia con Neuroticismo y Extraversión (negativa) y la manía con Neuroticismo, Extravesión y Amabilidad (negativa) [19], inplicando también cambios en los sesgos cognitivos y perceptivos [20].

Así, si consideramos al trastorno bipolar como una dimensión única, sería la Extraversión (del FGP) el predictor del trastorno. Eso significa que las alternancias que observamos en la Figura 1 representan la activación y caída extremas de los extravertidos (quienes puntúan más alto en el CFGP). Los puntos más altos y los más bajos representarían los puntos de bifurcación.

Por tanto, el estudio de la personalidad de los pacientes con trastorno bipolar, de la evolución de del trastorno en el tiempo, y de los fundamentos biológicos del mismo así como los mecanismos de acción de los fármacos y de las psicoterapias aplicadas, podrán en un futuro aportar valiosas contribuciones a una teoría 
integradora de la personalidad, cerebro y enfermedad mental.

\section{Conclusiones}

Nuestro modelo dinámico de personalidad ha sido capaz de explicar los cambios y las reacciones de la personalidad total (Factor General de Personalidad) a determinados estímulos, como las drogas, tanto a corto como a largo plazo, lo que ha permitido elaborar un modelo de adicción a las cocaína [11].

Ahora bien, nuestro modelo aspira a ser un modelo general de personalidad que explique no solo los mecanismos básicos del comportamiento humano, sino también del surgimiento de las patologías.

En el paso de lo "normal” a lo "anormal”, el sistema de estrés, sustrato biológico del FGP, juega un papel crucial. Un nivel crónico de estrés desencadena tanto trastornos mentales como problemas de salud física. Cuando un extravertido se ve sometido a altos niveles de estrés, desarrollará síntomas psicóticos y reacciones agresivas, mientras que los introvertidos desarrollarán trastornos neuróticos. Si la presión sobre el sistema de estrés aumenta, los extravertidos presentarán síntomas de tipo neurótico (obsesiones, compulsiones, ansiedad) mientras que los introvertidos pueden experimentar síntomas psicóticos. Esto se debe a que la inhibición transmarginal se produce en fases distintas para extravertidos e introvertidos.

Con lo que acabamos de exponer, podemos ver que hemos abarcado todos los trastornos clínicos, tanto del espectro de las neurosis como de las psicosis, basándonos en mecanismos que ya fueron predichos por la TRUP [4]. El reto que nos planeamos ahora es modelar matemáticamente esa transición de lo normal a lo anormal, para lo cual proponemos modelos no lineales y espacio-temporales que expliquen la disrupción que supone el surgimiento de un síntoma patológico en contraste con el comportamiento normal del ser humano. El caso de la Figura 1 reproduciría una respuesta anormal, propia de un trastorno bipolar.

El desarrollo de un modelo no lineal o espaciotemporal puede ser el que finalmente permita desvelar los mecanismos fundamentales del comportamiento humano y de los trastornos mentales.

\section{REFERENCIAS}

[1] Trull, T.J. and Sher, K.J. (1994). Relationship between the five-factor model of personality and Axis I disorders in a nonclinical simple. Journal of Abnormal Psychology, 103, 350-360.

[2] Millon, T. y Everly, G.S. (1994). La Personalidad y sus trastornos. Barcelona: Martínez Roca.

[3] Eysenck, H. J. (1967). The Biological Basis of Personality. Springfield, MA: C. C, Thomas.

[4] Amigó, S. (2005). La teoría del rasgo único de personalidad. Hacia una teoría unificada del cerebro y la conducta [The Unique Personality Trait Theory. Towards a unified theory of brain and behavior]. Valencia, Spain: Universitat Politècnica de València.

[5] Amigó, S., Caselles, A. and Micó, J.C. (2010). General Factor of Personality Questionnaire (GFPQ). Only one factor to understand personality? Spanish Journal of Psychology, 13, 5-17.

[6] Amigó S., Micó J. C., and Caselles A. (2009). Five adjectives to explain the whole personality: A brief scale of personality. Revista Internacional de Sistemas, 16, 4143.

[7] Caselles A, Micó, C, and Amigó S. Dynamics of the General Factor of Personality in response to single dose of caffeine. The Spanish Journal of Psychology, 14 (2011): 675-692.

[8] Amigó S, Caselles A, Micó JC and García J.M. Dynamics of the unique trait of personality: blood's glutamate in response to methylphenidate and conditioning. Revista Internacional de Sistemas, 16 (2009): 35-40.

[9] Amigó S, Caselles A and Micó J. C. Self-regulation therapy to reproduce drug effects: A suggestion technique to change personality and DRD3 gene expression. The International Journal of Clinical and Experimental Hypnosis 61 (2013): 282-304.

[10] Micó JC, Amigó S and Caselles A. Changing the General Factor of Personality and the c-fos expression with methylphenidate and Self-Regulation Therapy. The Spanish Journal of Psychology, 15(2) (2012): 850-867.

[11] Caselles, A., Micó, J. C., and Amigó, S. (2010). Cocaine addiction and personality: A mathematical model. British Journal of Mathematical and Statistical Psychology, 63, 449-480.

[12] Micó J. C., Amigó S., and Caselles A. (2014). From the Big Five to the General Factor of Personality: a Dynamic Approach. Spanish Journal of Psychology, 17, $1-18$. 
[13] Eysenck, H. J., and Eysenck, S. B. G. (1985). Eysenck Personality Questionnaire (EPQ-R) and Short Scale (EPQ-RS). London: Hodder \& Stoughton.

[14] Gray, J.A. (1993). La psicología del miedo y el estrés. Barcelona: Labor.

[15] Micó, J. C. and Sanz, M. T. (2013). A spatiotemporal approach to brain dynamics, Revista Internacional de Sistemas, 18, 29-38 (Available in http://www.uv.es/caselles/).

[16] Lewis, M., Scott, J. and Frangou, S. (2009). Impulsivity, personality and bipolar disorder. European Psychiatry, 24, 464 - 469.

[17] Sariusz-Skapska, M., Czabala, C., Dudek, D., Zieba, A. and Rduch, S. (2003). [Personality traits in patients with unipolar and bipolar disorder] [Polish] Psychiatr. Pol,. 37, 225-233.

[18] Smillie, L.D., Bhairo, Y., Gray, J., Gunasinghe, C., Elkin, A., McGuffin, P. and Farmer, A. (2009). Personality and the bipolar spectrum: normative and classification data for the Eysenck Personality Questionnaire - Revised. Compr. Psychiatry, 50, 48-53.

[19] Quilty, L.C., Sellbon, M., Tackett, J.L. and Bagby, R.M. (2009). Personality trait predictors of bipolar disorder symptoms. Psychiatry Research, 169, 159-163.

[20] García-Blanco, A. C., Perea, M. and Livianos, L. (2013). Mood-congruent bias and attention shifts in the different episodes of bipolar disorder. Cognition and Emotion, 27, 1114-1121. 